\title{
Driving force profile design in comb drive electrostatic actuators using a level set-based shape optimization method
}

\section{$\operatorname{AUTHOR}(S):$}

Kotani, Takayo; Yamada, Takayuki; Yamasaki, Shintaro; Ohkado, Makoto; Izui, Kazuhiro; Nishiwaki, Shinji

\section{CITATION:}

Kotani, Takayo ...[et al]. Driving force profile design in comb drive electrostatic actuators using a level set-based shape optimization method. Structural and Multidisciplinary Optimization 2014, 51(2): 369-383

\section{ISSUE DATE:}

2014-07-20

URL:

http://hdl.handle.net/2433/198731

\section{RIGHT:}

The final publication is available at Springer via http://dx.doi.org/10.1007/s00158-0141130-y.; 許諾条件により本文ファイルは2015-07-20に公開.; This is not the published version. Please cite only the published version.; この論文は出版社版でありません。引用 の際には出版社版をご確認ご利用ください。 


\title{
Driving force profile design in comb drive electrostatic actuators using a level set-based shape optimization method
}

\author{
Takayo Kotani · Takayuki Yamada - Shintaro Yamasaki · Makoto Ohkado · \\ Kazuhiro Izui · Shinji Nishiwaki
}

Received: date / Accepted: date

\begin{abstract}
Electrostatic actuators, actuators actuated by electrostatic forces, are now widely used as sensors and switches, especially in Micro-Electro-Mechanical Systems (MEMS). Among different kinds of electrostatic actuators, the comb drive type is one of the most popular because it has a relatively large range of displacement.

In design problems for electrostatic actuators, the driving force profile is of primary engineering importance. In this paper, we develop a structural optimization method for comb drive electrostatic actuators that achieves prescribed driving force profiles, based on a level set-based shape optimization method that provides optimal configurations with clear boundaries, solutions that are valid in an engineering sense.
\end{abstract}

Accurate calculation of the electrostatic forces that occur on the structural boundaries during optimization is important for developing actuators that operate with prescribed driving forces. In the conventional level set-based shape optimization methods, inaccuracies in the calculation of these electrostatic forces occur because the structural boundaries are seldom aligned with the finite element method (FEM)

\section{T. Yamada}

Department of Mechanical Engineering and Science, Kyoto University, C3, Kyotodaigaku-katsura, Nishikyo-ku, Kyoto, 615-8540 Japan

Tel: +81-75-383-3600

Fax: +81-75-383-3601

E-mail: takayuki@me.kyoto-u.ac.jp

T. Kotani $\cdot$ K. Izui $\cdot$ S. Nishiwaki

Department of Mechanical Engineering and Science, Kyoto University, C3, Kyotodaigaku-katsura, Nishikyo-ku, Kyoto, 615-8540 Japan

S. Yamasaki

Department of Mechanical Engineering, Osaka University,

2-1 Yamadaoka, Suita, Osaka, 565-0871 Japan

M. Ohkado

Information \& Electronics Research Division, Toyota Central R\&D Labs., Inc.,

41-1 Yokomichi, Nagakute, Aichi, 480-1192 Japan nodes. To precisely calculate the electrostatic forces, we developed a mesh adaptation scheme by which the finite element nodes are brought into alignment with the structural boundaries at every iteration of the optimization procedure.

In the following, we explain the details of the proposed level set-based shape optimization method, in which a multiobjective optimization problem is formulated to achieve a prescribed driving force profile. The sensitivity is derived using the adjoint variable method. Four numerical examples are provided, to examine the suitability of the proposed optimization method.

Keywords Shape Optimization · Level Set Method . Electrostatic Actuators · Comb Drive

\section{Introduction}

Electrostatic actuators have been increasingly deployed, especially in MEMS. The operating principle of electrostatic actuators is based on the electrostatic forces that occur on electrode surfaces. This principle has been known for hundreds of years, but widespread use of such devices is a recent phenomenon because the voltage requirements are unrealistically high for large devices. At the MEMS scale, however, the voltage requirements are reasonably small and the simplicity of electrostatic actuator structures allows manufacturing using well-established micro-processing technologies developed in semiconductor industries. Thus, electrostatic actuators are currently one of the most popular type of actuators in the MEMS field.

There are different types of electrostatic actuators, including comb drive (Legtenberg et al, 1996; Ye et al, 1998; Ye and Mukherjee, 1999; Grade et al, 2003), cantilever (Kim et al, 1996; Ou et al, 2011), and parallel plate (Nemirovsky and Bochobza-Degani, 2001; Seeger and Boser, 2003), used in many different micro-devices such as switches, sensors, 
and electronic filters. Among the various kinds of electrostatic actuators, the comb drive type has a relatively large range of displacement and is near the top in terms of popularity. Here, we focus on the structural optimization of comb drive electrostatic actuators.

When designing comb drive electrostatic actuators, much effort is focused on obtaining a structure that achieves a specific driving force profile. The driving force profile is the primary determinant of comb drive electrostatic actuator performance. The driving force profile of typical comb drive electrostatic actuators is constant during most of the displacement range, except near the limits of the range. A comb drive actuator is always paired with a spring mechanism that has a linear response except at large displacements, when the response becomes nonlinear. Differences between the comb and spring response curves make it difficult to design actuators that provide an intended performance. Therefore, it would be ideal if it were possible to design the driving force profile of a comb drive actuator, for example, to arbitrarily obtain a linear or quadratic response for the driving forces. Being able to design actuators with prescribed driving force profiles is thus of primary engineering importance.

Driving force profiles are determined by the shapes of the electrodes. Obtaining appropriate electrode shapes by trial and error methods is difficult because simultaneous consideration of multiple governing equations, applied to the electrodes at different relative positions, is required. Here, a structural optimization method can play an important role in finding optimal electrode shapes for electrostatic actuators.

There are several previous studies of structural optimization methods for electrostatic actuators. Shape optimization of comb drive electrostatic actuators has been explored by Ye et al (1998; 1999), who used a polynomial functionbased shape optimization and obtained optimal structures that successfully minimized the objective function. However, during the optimization, shapes could only deform within the range available with the polynomial function, so significant changes in shape were impossible.

Topology optimization (Bendsøe and Kikuchi, 1988), on the other hand, allows large structural changes. Topology optimization of electrostatic actuators has been studied by Raulli et al. (2005), Alwan et al. (2006a; 2006b), and Yoon et al. (2008), who collectively dealt with cantilever and inverter electrostatic actuator types. They successfully obtained optimal shapes that achieved target displacements. However, these methods, based on the use of the Solid Isotropic Material with Penalization (SIMP) method (Bendsøe, 1989; Yang and Chuang, 1994; Bendsøe and Sigmund, 1999), were affected by the problem of grayscales that often occurs in SIMP methods, hence the structural boundaries were not entirely clear. Some optimal configu- rations also included hinges, which are inappropriate from a manufacturing point of view.

To deal with the above problems, Qian et al. (2013) proposed a SIMP optimization method for electrostatic actuators using a robust filter that does provide clear structural boundaries and controls the minimum width of the obtained structures. The robust filter in the optimization procedure selects the structure that offers the least displacement from the so-called blue-print, under-etched, and over-etched structures, and maximizes the displacement of the chosen structure in each iteration. They successfully obtained optimal designs with clear boundaries for inverter type electrostatic actuators. Their method, however, aimed to maximize the force applied to the output port, whereas our method, as discussed below, seeks to obtain a structure that achieves a prescribed driving force profile.

Level set-based structural optimization methods, pioneered in 2000 (Sethian and Wiegmann, 2000), provide optimal configurations that are free from grayscales. Level setbased methods express structural boundaries by taking the iso-surface of the level set function. During the optimization procedure, the changes in the structural boundaries are expressed by the changes in the iso-surface of level set function.

Wang et al. (Wang et al, 2003) and Allaire et al. (Allaire et al, 2004) independently proposed structural optimization methods in which the Hamilton-Jacobi equation is used for updating the level set function, to move the structural boundaries. On the other hand, Yamada et al. (Yamada et al, 2010, 2011) proposed a different type of method in which a reaction-diffusion equation is used for updating the level set function, a scheme that can allow topological changes in the target structures. Comparing these methods, the former can be categorized as shape optimization, whereas the latter can be categorized as topology optimization, and in this paper, we construct a structural optimization method for the design of electrostatic actuators using a level set-based shape optimization method. Our level set-based shape optimization method allows for the disappearance and integration of holes, and the cleaving of a material domain into two separate material domains, but does not allow the creation of holes. This will not affect the optimization of comb drive electrostatic actuators here because the electrostatic forces only occur on the structural surfaces, and performances are not improved by the creation of holes inside the structure.

When optimizing electrostatic actuators, accurate calculation of electrostatic forces is important so that actuators achieve prescribed driving force profiles. In level set-based shape optimization methods, a new approach is required in the calculation of the electrostatic forces because the structural boundaries, on which the electrostatic forces occur, move during the calculation and seldom match to the nodes 
of the finite element mesh. This impedes accurate calculation of the electrostatic forces on the boundaries.

Several methods have been proposed to match the FEM nodes with the moving structural boundaries. One of the most popular methods is the Arbitrary Lagrangian Eulerian (ALE) method (Huerta and Liu, 1988; Hirt et al, 1997) which is frequently used in fluid dynamics. The ALE method precisely describes the moving boundaries and interfaces, using an arbitrary movement of a reference frame. The computation, however, is not very robust with this method when the boundary movement is complex. Another major method which tracks the changing boundaries is the Extended Finite Element Method (X-FEM) (Belytschko and Black, 1999; Dolbow and Belytschko, 1999; Sukumar et al, 2001), widely used for tracking cracks in structures. The XFEM does not require that new or moving internal boundaries be meshed, but an additional function, called the enrichment function, is required to model new or moving internal boundaries during calculation, and complex calculation algorithms are needed.

To track level set boundaries in a simple manner in our research, for electrostatic actuator optimization problems, we developed a mesh adaptation scheme originally proposed by Yamasaki et al. (2011). In this scheme, the FEM nodes close to the level set boundaries are moved to positions on the level set boundaries. This method does not require any changes in the optimization algorithm or additional functions, and can accurately track level set boundaries that undergo large changes. To avoid extreme distortion of element shapes during the mesh adaptation, Laplacian smoothing is employed to reposition FEM nodes other than those moved to the structural boundaries.

Based on the mesh adaptation scheme mentioned above, we developed a level set-based shape optimization method for comb drive electrostatic actuators. Our method allows large structural changes and maintains clear boundaries during the optimization. A multi-objective functional is formulated in order to obtain an optimal configuration that achieves a prescribed driving force profile. The sensitivity is obtained by the adjoint variable method, and the derivation is described in Section 3.2. The electrostatic forces that occur on the structural boundaries are calculated using Maxwell's stress tensor. In the numerical examples, we optimize four comb drive electrostatic actuator design examples and present optimal configurations that achieve prescribed driving force profiles.

The level set-based shape optimization method and the details of the electrostatic actuator optimization problem formulation are explained in Section 2. We describe the numerical implementation in Section 3 and the numerical examples are provided in Section 4. The suitability of the method is also discussed in Section 4, and Section 5 provides a summary.

\section{Formulations}

\subsection{Level set-based shape optimization method}

In this subsection, the level set-based shape optimization method is briefly explained. In this method, the optimization problem is solved using a time evolutional equation that provides optimal solution candidates.

We consider the shape optimization of a material domain $\Omega$ in the fixed design domain $D$ and we introduce the level set function $\phi(x)$, a scalar function. The function $\phi(x)$ is a signed distance function (Osher and Fedkiw, 2003) representing distances from the structural boundaries. The material domain $\Omega$ and the material boundary, i.e., $\partial \Omega$, are expressed by this level set function. As shown in (1), values of $\phi$ greater than zero indicate elements in the material domain, while values of $\phi$ less than zero indicate elements in the void domain. The zero iso-surface of the level set function expresses the structural boundaries.

$$
\begin{cases}\phi(\boldsymbol{x})>0 & \text { for } \quad \forall \boldsymbol{x} \in \Omega \backslash \partial \Omega \\ \phi(\boldsymbol{x})=0 & \text { for } \forall \boldsymbol{x} \in \partial \Omega \\ \phi(\boldsymbol{x})<0 & \text { for } \forall \boldsymbol{x} \in D \backslash \Omega\end{cases}
$$

where $\boldsymbol{x}$ represents a point in the design domain.

Changes in the structural shape occur during optimization as the level set function is updated. We now introduce a fictitious time $t$, and the time derivative of the level set function yields the Hamilton-Jacobi equation (Wang et al, 2003; Allaire et al, 2004), as follows.

$$
\frac{\partial \phi(\boldsymbol{x}, t)}{\partial t}+\frac{\mathrm{d} \boldsymbol{x}}{\mathrm{d} t} \cdot \nabla \phi(\boldsymbol{x}, t)=0
$$

With the velocities of structural boundary movement in the normal direction denoted by $V_{N}(\boldsymbol{x}, t)$ and the normal direction vector denoted by $\boldsymbol{n}_{\phi}$, the following relation is obtained.

$$
\frac{\mathrm{d} \boldsymbol{x}}{\mathrm{d} t}=V_{N}(\boldsymbol{x}, t) \boldsymbol{n}_{\phi}
$$

$\boldsymbol{n}_{\phi}$ is now defined as follows.

$\boldsymbol{n}_{\phi}=\nabla \phi /|\nabla \phi|$

From (3) and (4), the Hamilton-Jacobi Equation (2) is now rewritten as

$$
\begin{aligned}
\frac{\partial \phi(\boldsymbol{x}, t)}{\partial t} & +V_{N}(\boldsymbol{x}, t) \boldsymbol{n}_{\phi} \cdot \nabla \phi(\boldsymbol{x}, t) \\
& =\frac{\partial \phi(\boldsymbol{x}, t)}{\partial t}+V_{N}(\boldsymbol{x}, t)|\nabla \phi(\boldsymbol{x}, t)|=0 .
\end{aligned}
$$

In our level set-based shape optimization method, the sensitivity, which is the design variable derivative of the objective functional, is applied to the velocities of the structural boundary $V_{N}(\boldsymbol{x}, t)$. 
The level set function is initially set to have the property of a signed distance function, but it looses this property when it is updated using (5). Therefore, the level set functions need to be re-initialized (Sussman et al, 1994; Sethian, 1999) after several iterations of the optimization procedure. We employ the geometry-based re-initialization scheme proposed by Yamasaki et al. $(2010 ; 2011)$. In this scheme, the signed distance function property is strictly maintained by re-initializing the level set function after each update of the level set function, so that $|\nabla \phi(x, t)|=1$ is maintained. Equation (5) can therefore be simplified as follows, which facilitates the updating of the level set function.

$\frac{\partial \phi(\boldsymbol{x}, t)}{\partial t}+V_{N}(\boldsymbol{x}, t)=0$

Solutions to the Hamilton-Jacobi equation are not always smooth, so a regularization method is required during the optimization process. The details of the regularization method are discussed in Section 3.2.

Note that the level set function $\phi(x)$ is discretized using the FEM. The details are explained in Section 3.

\subsection{Governing equations}

A comb drive electrostatic actuator consists of two combshaped electrodes, as shown in Fig. 1. One electrode is

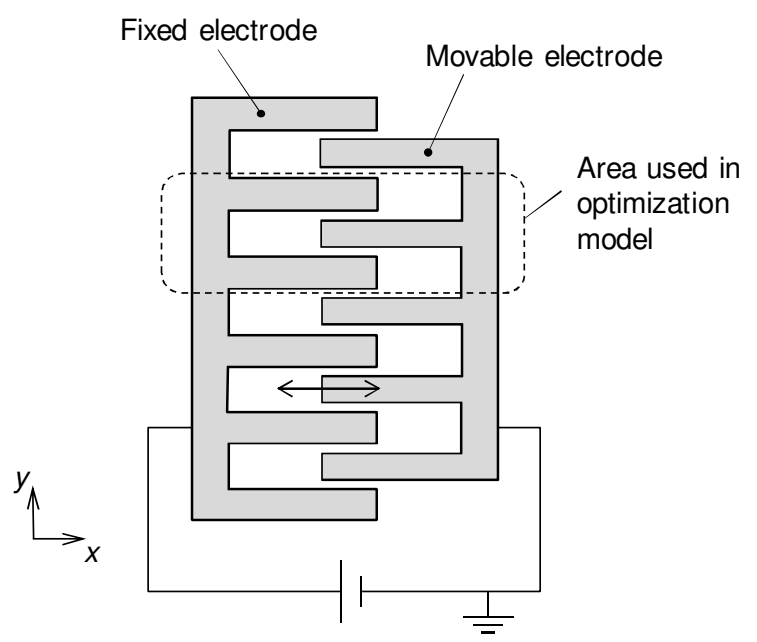

Fig. 1 Comb drive electrostatic actuator model

grounded and the other electrode has an electrical voltage imposed. The grounded electrode moves toward the other electrode when a voltage is applied and the distance between the two electrodes is reduced. This movement is the basis for the use of comb drive electrostatic devices as actuators.

One advantage of comb drive electrostatic actuators is that their displacement range can be relatively large, given an appropriate design and length of the teeth of the two electrodes. The primary concern of engineers designing comb drive actuators is to define the driving force as a function of displacement, for example, to obtain a device that has a linear driving force profile. Here, we formulate the optimization problem for comb drive electrostatic actuators to achieve pre-defined driving force profiles.

Electrostatic actuators operate when electrostatic forces are imposed on electrode surfaces by an electrostatic field, so the equations that describe the electrostatic field must be solved in order to optimize the design of an electrostatic actuator.

We set a design domain $D$ as shown in Fig. 2. The fixed

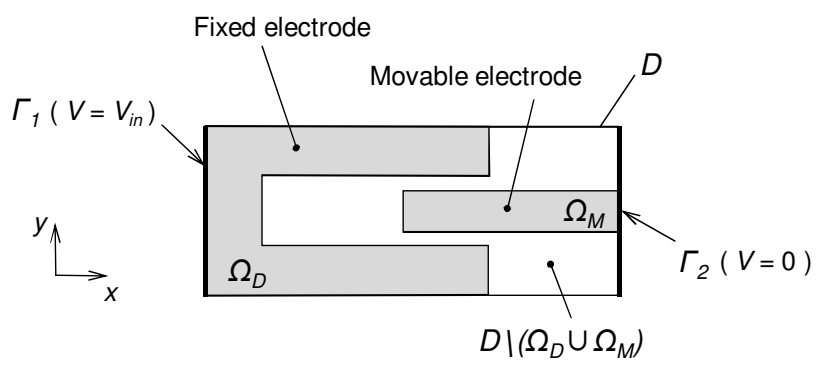

Fig. 2 Design domain and boundary conditions

electrode, a material domain, is denoted $\Omega_{D}$, and the movable electrode, also a material domain, is denoted $\Omega_{M}$. We used subscript $D$ when denoting the fixed electrode $\Omega_{D}$ because the shape of the fixed electrode is the design target in the optimization problem that is discussed below. Both electrodes are made from a conductive material. The void domain, which is air, is denoted $D \backslash\left(\Omega_{D} \cup \Omega_{M}\right)$.

Comb drive electrostatic actuators used in real-world applications have multiple pairs of teeth in order to create larger driving forces, as depicted in Fig. 1. In our study, to simplify the optimization model, we confine our design to one set of teeth, as shown in Fig. 2. The driving force of a design consisting of numerous sets of teeth can be obtained simply by multiplying the driving force of a single set by the number of sets.

We assume that there is no external electric charge or magnetic field. Extracting the relevant terms from Maxwell's equations, the governing equation is defined in the void domain as follows, using the vacuum permittivity $\varepsilon_{0}$ and the electric potential $V$.

$\boldsymbol{\nabla} \cdot\left(\varepsilon_{0} \nabla V\right)=0 \quad$ in $\quad D \backslash\left(\Omega_{D} \cup \Omega_{M}\right)$

In our method, this governing equation is extended to the entire design domain $D$ by introducing a sufficiently large fictitious permittivity to the material domain. That is, the 
governing equation is approximately solved in domain $D$, which is analogous to the ersatz material approach (Allaire et al, 2004; Yamada et al, 2011). Hence, the governing equation is redefined as below.

$$
\boldsymbol{\nabla} \cdot(\varepsilon(\phi) \nabla V)=0 \quad \text { in } \quad D,
$$

where $\varepsilon(\phi)$ is the extended permittivity, defined in the entire design domain $D$.

The boundary $\Gamma_{1}$, which has contact with the fixed electrode, is charged at electric potential $V_{\text {in }}$ and the boundary $\Gamma_{2}$, which has contact with the movable electrode, is connected to ground as shown in Fig. 2. The boundary conditions are follows.

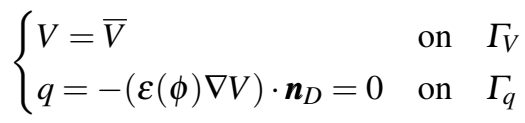

where $\bar{V}$ is the prescribed electric potential $V$ on boundary $\Gamma_{V}=\Gamma_{1} \cup \Gamma_{2}, \Gamma_{q}=\partial D \backslash \Gamma_{V}$ has a Neumann boundary condition imposed, and $\boldsymbol{n}_{D}$ is the normal unit vector on boundary $\partial D$. The governing equation (8) is now rewritten in the weak form using the principle of virtual work.

$\int_{D} \nabla \hat{V} \cdot(\varepsilon(\phi) \nabla V) \mathrm{d} \Omega=0$,

where $\hat{V}$ is the test function. Equation (10) is rewritten using the electric field vector $\boldsymbol{E}=-\boldsymbol{\nabla} V$, as follows.

$\int_{D} \hat{\boldsymbol{E}} \cdot(\varepsilon(\phi) \boldsymbol{E}) \mathrm{d} \Omega=0$

\subsection{Formulations for shape optimization}

We aim to develop an optimization method for comb drive electrostatic actuators so that the optimal structure realizes a prescribed driving force profile. We optimize the shape of the fixed electrode in the optimization problem in this paper.

We define the target driving force $\boldsymbol{T}^{j^{*}}$ at discrete positions of the movable electrode, defined as $j=1, \cdots, m$, as shown in Fig. 3, with $m$ arbitrarily defined. The actual driving force at position $j$ is calculated by taking the boundary integral of electrostatic force $\boldsymbol{t}^{j}$ on the boundary of the fixed electrode, denoted $s$. The electrostatic force is calculated using Maxwell's stress tensor defined as follows:

$$
\begin{aligned}
\boldsymbol{t}(\boldsymbol{\nabla} V) & =\boldsymbol{t}(\boldsymbol{E}) \\
& =-\varepsilon_{0}\left\{\boldsymbol{E} \otimes \boldsymbol{E}-\frac{1}{2}(\boldsymbol{E} \cdot \boldsymbol{E}) \boldsymbol{I}\right\} .
\end{aligned}
$$

We note that only the terms pertaining to the electrostatic field are extracted for our calculation. To achieve the target driving force at position $j$, the objective functional $F^{j}$ is defined as the square of the differences between the target

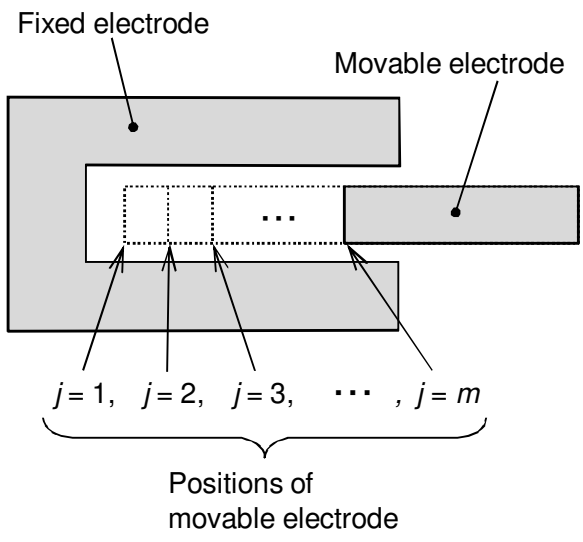

Fig. 3 Movable electrode's positions

driving force $\boldsymbol{T}^{j *}$ and the actual driving force $\boldsymbol{T}^{j}$, as shown below, and this objective functional is to be minimized.

$$
\begin{aligned}
\inf _{\phi} \quad F^{j} & =\left\{\boldsymbol{T}\left(\nabla V^{j}, \phi\right)-\boldsymbol{T}^{j *}\right\}^{2} \\
& =\left(\int_{s} \boldsymbol{t}\left(\boldsymbol{\nabla} V^{j}\right) \cdot \boldsymbol{n}_{s}(\phi) \mathrm{d} \Gamma-\boldsymbol{T}^{j^{*}}\right)^{2},
\end{aligned}
$$

where $s$ is the boundary of an electrode in a comb drive electrostatic actuator and $\boldsymbol{n}_{s}$ is a unit normal vector on boundary $s$. We note that the definition of the objective functional that satisfies the design requirement is not unique.

To obtain a configuration that achieves the prescribed driving force profile, we formulate the optimization problem as a multi-objective optimization problem. We formulate a multi-objective functional $F$ consisting of $F^{j}$ and simultaneously minimize the differences between target driving forces and actual driving forces at multiple positions $j$. To obtain a good convergence performance during the optimization process, we use the logarithm of the summation of the exponential of $F^{j}$ for the objective functional $F$. The optimization problem is formulated as follows.

$$
\begin{aligned}
& \inf _{\phi(\boldsymbol{x})} \quad F[\boldsymbol{\nabla} V, \phi]=\log \left\{\sum_{j=1}^{m} \exp \left(w^{j} F^{j}\right)\right\} \\
& \text { subject to } \\
& \begin{array}{ll}
\boldsymbol{\nabla} \cdot\left(\varepsilon(\phi) \nabla V^{j}\right)=0 & \text { in } \quad D \\
V^{j}=\bar{V} & \text { on } \Gamma_{V} \\
q^{j}=-\left(\varepsilon(\phi) \nabla V^{j}\right) \cdot \boldsymbol{n}_{D}=0 & \text { on } \Gamma_{q}
\end{array}
\end{aligned}
$$

where $w^{j}$ is a weighting function. This objective functional increases the impact of the sensitivity of the $j$-th position that has the largest difference between the target driving force and the actual driving force. The range of $w^{j}$ must be appropriately defined, since values of $w^{j}$ that are too large 
may cause memory overflow. The derivation of the sensitivity is explained in Section 3.2.

Note that in electrostatic actuator optimization problems, a volume constraint is relatively unimportant since these devices are MEMS-scale and there is generally little need to reduce their volume. We therefore do not employ a volume constraint. Additionally, to avoid situations where the two electrodes may stick together, we define a thin non-design domain that surrounds the fixed electrode. By appropriately defining design and non-design domains, boundary limits are provided implicitly. This is one advantage of our method, which uses an Eulerian coordinate system, compared to general shape optimization methods that use a Lagrangian coordinate system and cannot define nondesign domains.

\section{Numerical implementations}

The flow of the optimization is shown in Fig. 4. First, the

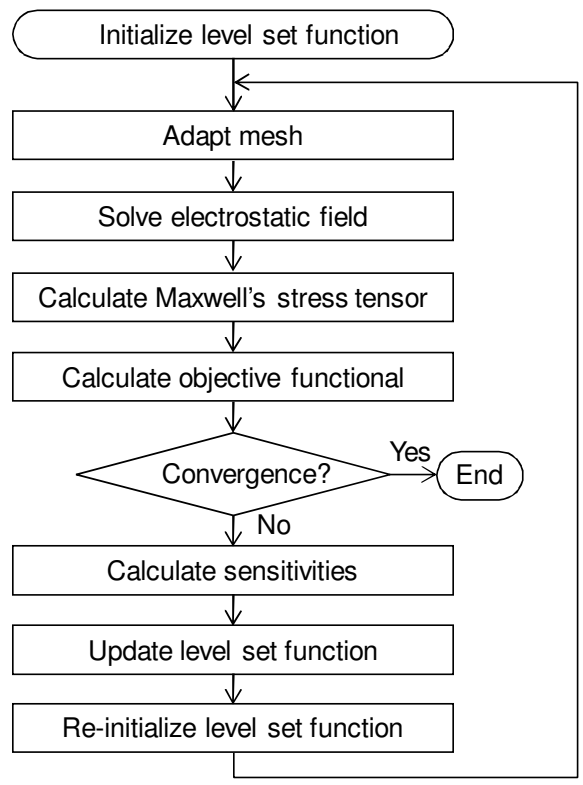

Fig. 4 Flowchart of optimization procedure

initial value of the level set function is set. The mesh adaptation is then carried out. The nodes that are close to but not on the level set boundaries are moved so that they lie on the boundaries. The details of this mesh adaptation scheme are discussed in Section 3.1. In the third step, the electrostatic field is solved by the FEM. In the fourth step, the electrostatic forces acting on the nodes on the structural boundaries are determined by calculating Maxwell's stress tensor.
Then, the objective functional is calculated and if it has converged, the optimization ends. If the optimization has not converged, the sensitivities are calculated and the level set function is updated according to the sensitivities. The level set function is discretized using the FEM and its value is given at each node. Hence, the level set function is updated using the following spatially-discretized equation.

$\boldsymbol{\Phi}^{t+\Delta t}=\boldsymbol{\Phi}^{t}-\Delta t \boldsymbol{V}_{N}^{t}$

$\boldsymbol{\Phi}^{t}$ represents the vector of the level set function value at each node at time $t$, and $\boldsymbol{V}_{N}^{t}$ represents the vector of velocity in the normal direction at each node at time $t . \Delta t$ is the time increment. At the end of the optimization procedure, the level set function is re-initialized using the geometrybased re-initialization scheme discussed in Section 2.1, and the procedure returns to the second step. The objective functional is thereby minimized and an optimal configuration is derived.

\subsection{Mesh adaptation scheme}

The forces driving an electrostatic actuator are the electrostatic forces that occur on the structural boundaries. To obtain an appropriate design, these electrostatic forces must be precisely calculated during the optimization process. Such calculations, however, are not straightforward in level setbased shape optimization methods because the FEM nodes seldom lie on the structural boundaries. To accurately calculate the forces on the structural boundaries, the position of the finite elements nodes and the structural boundaries need to be congruent.

In this study, we further develop the mesh adaptation scheme originally proposed by Yamasaki et al. (2011), extending it to electrostatic actuator optimization problems. The nodes in the void domain that are close to the structural boundaries are moved so that they lie precisely on the level set boundaries. When only the nodes close to the boundaries are moved, certain element shapes become distorted, which lowers mesh quality and therefore degrades the accuracy of the analysis. To mitigate this problem, we use a Laplacian smoothing method.

The formulation of our mesh adaptation scheme is as follows. The movement vectors of the nodes, i.e., displacements from original node positions to new positions, are represented as $\boldsymbol{d}_{x}, \boldsymbol{d}_{y}$, and $\boldsymbol{d}_{z}$. The mesh adaptation is executed by updating the node coordinates according to these movement vectors, as in (20).

$\left\{\begin{array}{l}\boldsymbol{X}=\boldsymbol{X}_{0}+\boldsymbol{d}_{x} \\ \boldsymbol{Y}=\boldsymbol{Y}_{0}+\boldsymbol{d}_{y} \\ \boldsymbol{Z}=\boldsymbol{Z}_{0}+\boldsymbol{d}_{z},\end{array}\right.$ 
where $\boldsymbol{X}, \boldsymbol{Y}$, and $\boldsymbol{Z}$ are the coordinates of all nodes after the mesh adaptation, and $\boldsymbol{X}_{0}, \boldsymbol{Y}_{0}$, and $\boldsymbol{Z}_{0}$ are the coordinates of all nodes before the mesh adaptation. The derivation of the movement vectors $\boldsymbol{d}_{x}, \boldsymbol{d}_{y}$, and $\boldsymbol{d}_{z}$ for each node is explained below.

Figure 5(a) shows the mesh structure before the mesh adaptation. Two-dimensional triangular elements are used

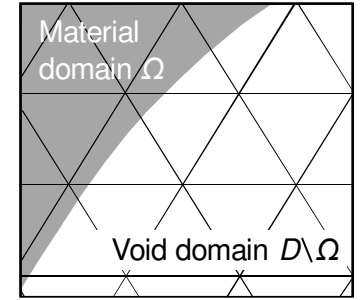

(a) Initial mesh

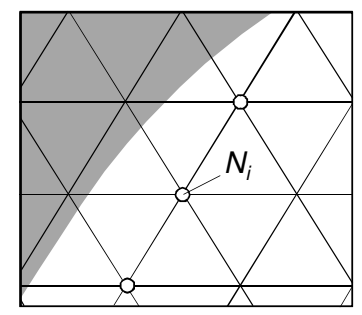

(c) Nodes $N_{i}$

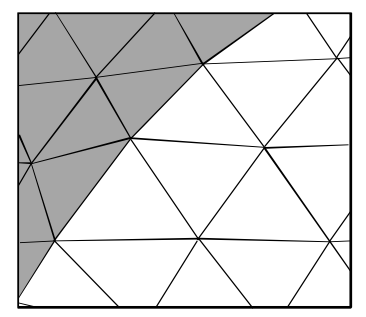

(e) Adapted mesh

Fig. 5 Mesh adaptation scheme

throughout Fig. 5, for the explanation of the mesh adaptation scheme, although the mesh adaptation scheme is a general scheme that is also valid for three-dimensional mesh structures. The gray areas represent the material domain $\Omega$ and the other areas represent the void domain. First, the elements that consist of both material and void are selected, and measurement points, denoted by black dots in Fig. 5(b), are populated at even intervals on the structural boundaries $\partial \Omega$ within each element. The nodes that exist in the void domain portion of the selected elements are denoted $N_{i}$, as shown in Fig. 5(c). As shown in Fig. 5(d), the $N_{i}$ nodes are moved to the closest measurement point on the structural boundary.
The components of the movement vectors $d_{x_{i}}, d_{y_{i}}$, and $d_{z_{i}}$ are calculated.

Next, the components of movement vectors for other nodes are calculated using the Laplacian smoothing method (Buell and Bush, 1973; Field, 1988). The movement vectors $\boldsymbol{d}_{x}, \boldsymbol{d}_{y}$, and $\boldsymbol{d}_{z}$ are obtained by solving the following equations, using the FEM.

$$
\begin{array}{lll}
\boldsymbol{K} \boldsymbol{d}_{x}=0 & \text { with } & d_{x_{i}}=\overline{d_{x_{i}}} \\
\boldsymbol{K} \boldsymbol{d}_{y}=0 & \text { with } & d_{y_{i}}=\overline{d_{y_{i}}} \\
\boldsymbol{K} \boldsymbol{d}_{z}=0 & \text { with } & d_{z_{i}}=\overline{d_{z_{i}}} \\
\boldsymbol{K}:=\bigcup_{e=1}^{n_{e}} \int_{A_{e}} \nabla^{\mathrm{T}} \boldsymbol{N} \nabla \boldsymbol{N} \mathrm{d} \Omega,
\end{array}
$$

where $\overline{d_{x_{i}}}, \overline{d_{y_{i}}}$, and $\overline{d_{z_{i}}}$ are the components of the movement vectors of the nodes that have been moved to the structural boundaries, calculated according to the procedure explained above. The total number of elements in design domain $D$ is represented as $n_{e}, \int_{A_{e}} * \mathrm{~d} \Omega$ is the domain integral of the $e$ th element, and $N$ is the shape function. Using the above procedure, the movement vectors $\boldsymbol{d}_{x}, \boldsymbol{d}_{y}$, and $\boldsymbol{d}_{z}$ for all the nodes are calculated, and mesh adaptation is completed as shown in Fig. 5(e).

When the curvature of the structural boundary is very large, the boundary arcs circularly with a central focus on a node, and this node then has multiple closest points at different locations on the boundary. In our method, to avoid this kind of situation, the structural boundaries are smoothed using a sensitivity filter, as discussed in Section 3.2. On the other hand, there are cases when multiple nodes share the same closest point on the boundary. In such cases, these nodes are excluded from the set of nodes that are forcibly displaced.

The mesh quality is examined using the following formula.

$q=\frac{4 \sqrt{3} g}{h_{1}^{2}+h_{2}^{2}+h_{3}^{2}}$,

where $q$ is the mesh quality index, $g$ is the area, and $h_{1}, h_{2}$ and $h_{3}$ are the side lengths of the triangular elements (Bank and $\mathrm{Xu}, 1996$; Bank and Smith, 1997). The value of $q$ ranges between 0 and 1 and an equilateral triangle has a $q$ value of 1. The quality of solutions is unaffected when $q$ is larger than 0.3 . The value of $q$ is computed for all elements at every iteration. In this manner, the mesh adaptation scheme enables accurate calculation of the electrostatic forces.

We note that the calculation cost of this scheme increases for three-dimensional problems, although it is not very high in two-dimension problems.

To calculate the electrostatic forces at the nodes on the structural boundaries, we need to select just the nodes that are on the boundaries. Any element with a side that contacts 
a structural boundary has two nodes for which the values of the level set functions are zero. These $\phi=0$ nodes are selected, and Maxwell's stress tensors at these selected nodes are calculated. Maxwell's stress tensors for nodes not on the structural boundaries are set equal to zero.

\subsection{Sensitivity analysis}

In this section, we derive the design sensitivities that will be used as $V_{N}$ in (6) when updating the level set function, and we use the adjoint variable method to derive these sensitivities. First, the boundary integral representing the driving force is replaced by the following domain integral by using level set function $\phi$ :

$\boldsymbol{T}\left(\boldsymbol{\nabla} V^{j}, \phi\right)=\int_{D} \boldsymbol{t}\left(\boldsymbol{\nabla} V^{j}\right) \overline{\boldsymbol{\delta}}(\phi) \mathrm{d} D$,

where $\overline{\boldsymbol{\delta}}(\phi)$ is defined using an approximated Heaviside function $H_{a}(\phi)$, as follows:

$\overline{\boldsymbol{\delta}}(\phi):=\frac{\nabla \phi}{|\nabla \phi|} \frac{\mathrm{d} H_{a}(\phi)}{\mathrm{d} \phi}$.

In our study, the Heaviside function is approximated as follows, since $H(\phi)$ is a step function.

$H_{a}(\phi)= \begin{cases}0 & (\phi<-h) \\ \frac{1}{2}+\frac{15}{16}\left(\frac{\phi}{h}\right)-\frac{5}{8}\left(\frac{\phi}{h}\right)^{3}+\frac{3}{16} & \left(\frac{\phi}{h}\right)^{5} \\ 1 & (-h \leq \phi \leq h) \\ & (h<\phi),\end{cases}$

where $h$ is the transition width between the material and void domains.

Next, the Lagrangian $\bar{F}$ is defined as follows:

$$
\begin{aligned}
& \bar{F}=\log \left[\sum_{j=1}^{m} \exp \left\{w^{j}\left(\int_{D} \boldsymbol{t}\left(\boldsymbol{\nabla} V^{j}\right) \overline{\boldsymbol{\delta}}(\phi) \mathrm{d} D-\boldsymbol{T}^{j *}\right)^{2}\right\}\right] \\
& +\sum_{j=1}^{m}\left[\int_{D} \hat{V}^{j} \boldsymbol{\nabla} \cdot\left(\varepsilon(\phi) \nabla V^{j}\right) \mathrm{d} D\right],
\end{aligned}
$$

where $\hat{V}^{j}$ represents the Lagrange multipliers. Furthermore, the adjoint system is defined as follows:

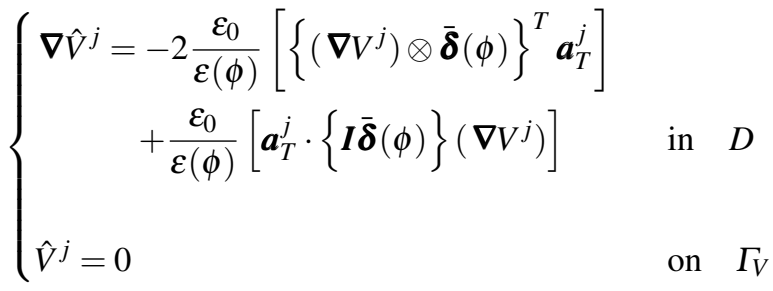

where coefficient vector $\boldsymbol{a}_{T}^{j}$ is defined in the following equation.

$\boldsymbol{a}_{T}^{j}:=\frac{2 w^{j}\left(\boldsymbol{T}^{j}-\boldsymbol{T}^{j *}\right) \exp \left\{w^{j}\left(\boldsymbol{T}^{j}-\boldsymbol{T}^{j *}\right)^{2}\right\}}{\sum_{j=1}^{m} \exp \left\{w^{j}\left(\boldsymbol{T}^{j}-\boldsymbol{T}^{j *}\right)^{2}\right\}}$

Thus, we obtain the following design sensitivity:

$$
\begin{gathered}
\delta \bar{F}=\sum_{j=1}^{m} \int_{D}\left\{\left(\frac{2 w^{j} W^{j}\left(\boldsymbol{T}^{j}-\boldsymbol{T}^{j *}\right)}{\sum W^{j}}\right) \cdot\left(\boldsymbol{t}\left(\boldsymbol{\nabla} V^{j}\right) \frac{\partial \overline{\boldsymbol{\delta}}(\phi)}{\partial \phi}\right)\right. \\
\left.-\left(\boldsymbol{\nabla} \hat{V}^{j}\right) \cdot\left(\frac{\partial \varepsilon(\phi)}{\partial \phi} \nabla V^{j}\right)\right\} \delta \phi \mathrm{d} D .
\end{gathered}
$$

Details concerning the derivation of the sensitivity are provided in the Appendix. We note that the first and second terms respectively represent the effects of geometrical changes and the effects of changes in Maxwell's stress tensor. Therefore, the first term can be neglected when changes in the shape are insignificant. We also note that the adjoint problem does not have to be solved, although the optimization problem is not a self-adjoint problem. That is, the design sensitivity is represented by the gradient of the Lagrange multiplier $\nabla \hat{V}$, and the adjoint system is constructed using the gradient of the Lagrange multiplier $\nabla \hat{V}$, since the objective functional is defined by using the domain integral of a function of a gradient of the state variable $V$.

As discussed in Section 2.1, the sensitivity $\frac{\delta F}{\delta \phi}$ is not necessarily smooth and thus needs to be regularized. We use a convenient Helmholtz type of filter for this regularization, chosen because it is a PDE filter that directly uses the FEM structure. The regularized sensitivity is denoted $\left(\frac{\delta F}{\delta \phi}\right)^{*}$. The regularization is carried out using the following equation.

$$
\left(\frac{\delta F}{\delta \phi}\right)^{*}+R^{2} \nabla^{2}\left(\frac{\delta F}{\delta \phi}\right)^{*}=\left(\frac{\delta F}{\delta \phi}\right),
$$

where $R$ is a parameter that affects the smoothness of the solutions. $\left(\frac{\delta F}{\delta \phi}\right)^{*}=\left(\frac{\delta F}{\delta \phi}\right)$ when $R=0$, and (32) is equivalent to Poisson's equation as $R \rightarrow \infty$.

This regularized sensitivity $\left(\frac{\delta F}{\delta \phi}\right)^{*}$ is used for the value of $V_{N}$ in (6) to update the level set function. The values of $V_{N}$ at nodes that are not on the structural boundaries are replaced with the $V_{N}$ values of the closest nodes that lie on the structural boundaries.

\section{Numerical Examples}

We first verify the reasonability of the mesh adaptation scheme using a parallel plate type of electrode, and then examine the suitability of our proposed optimization method using a comb drive electrostatic actuator model. 
4.1 Verification of mesh adaptation scheme reasonability

In this section, we verify the reasonability of the mesh adaptation scheme proposed in Section 3.1. The electrostatic force of an infinite parallel plate type of electrode can be solved analytically, but an infinite parallel plate cannot be solved by the FEM. Therefore, we use a sufficiently large parallel plate type of electrode, using electrodes that are $1 \mu \mathrm{m}$ thick, $42 \mu \mathrm{m}$ wide, and spaced $8 \mu \mathrm{m}$ apart. A voltage of $25 \mathrm{~V}$ is applied between the two electrodes. An evaluation region $20 \mu \mathrm{m}$ wide and $8 \mu \mathrm{m}$ high is set at the center of electrodes (Fig. 6). The region has a depth of $1 \mathrm{~m}$. The

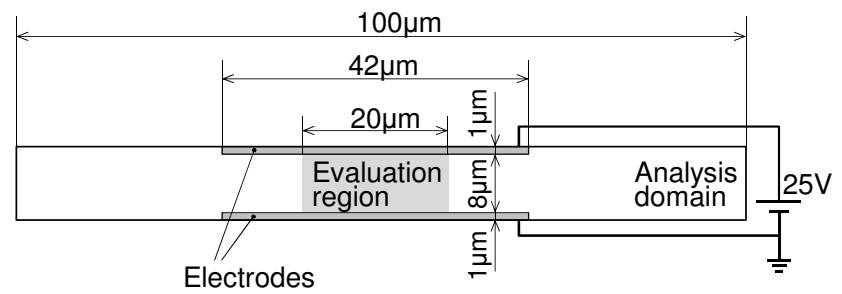

Fig. 6 Parallel plate model

material properties used in this problem are the permittivity of the void and material domains. The vacuum permittivity of the void domains is $8.85 \times 10^{-12} \mathrm{Fm}^{-1}$, and that of material domain is defined as being $10^{3}$ times larger, which is sufficiently larger than that of the void domain. The material property is approximated in the same manner as in the ersatz material approach, but we note that our method provides clear boundaries whereas the ersatz material approach does not. We solve the absolute value of the electrostatic forces occurring on the electrode surfaces within the evaluation region using an adapted mesh structure realized by our proposed method. For comparison, these forces are also solved analytically and by using a non-adapted mesh structure obtained by an ersatz material approach, a conventional approach that avoids re-meshing when using a level set method.

The electrostatic forces in Cases 1, 2, and 3 are respectively solved analytically, by using the adapted mesh obtained via our proposed mesh adaptation scheme, and by using the non-adapted mesh obtained via the ersatz material approach (Table 1). The analytical (Case 1) and adapted

Table 1 Electrostatic forces [N]

\begin{tabular}{ccc}
\hline Case1 & Case2 & Case3 \\
\hline $8.65 \times 10^{-4}$ & $8.72 \times 10^{-4}$ & $4.74 \times 10^{-6}$ \\
\hline
\end{tabular}

mesh (Case 2) solutions are nearly equal, but electrostatic force provided by the non-adapted mesh structure (Case 3) was much less than that of the other solutions. The electrostatic force is undervalued in Case 3 due to underevaluation of the electric field vector $\boldsymbol{E}$ because the material properties have intermediate values between those of the material and void domains around the structural boundaries on which the electrostatic forces occur. From above results, we confirm that the electrostatic force is appropriately obtained using our proposed mesh adaptation scheme. Furthermore, the ersatz material approach in which the mesh is not adapted to the boundaries does not provide accurate analysis of the electrostatic forces, and thus is not ideal for solving electrostatic actuator optimization problems.

\subsection{Numerical examples of proposed optimization method}

The model considers a single set of teeth, as shown in Fig. 7, although the fixed and movable electrodes in actual devices have numerous sets of teeth deployed in a line.

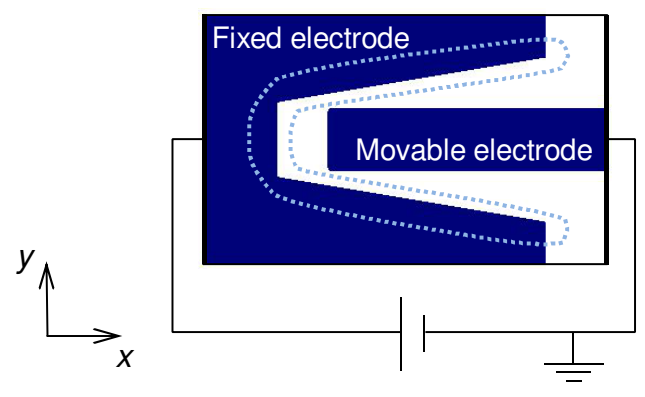

Fig. 7 Comb drive model for numerical examples

In our examples, we only optimize the shape of the fixed electrode because we determined that this approach was reasonable from the standpoint of calculation cost and the manufacturability of the comb drive designs, and it led to structures that achieve prescribed driving forces. The driving force is calculated by taking the boundary integral of the electrostatic forces on the surface of the fixed electrode along the length shown circled by the dotted line in Fig. 7.

We set the size of the design domain as $650 \times 400 \mu \mathrm{m}$, with a thickness of $10 \mu \mathrm{m}$. The initial configuration of the electrodes are shown in Fig. 8. An external voltage $V_{i n}=$ $100 \mathrm{~V}$ is applied to the fixed electrode and the movable electrode is grounded. Concerning the weighting function $w^{j}$ in (??), we assign a value of 1 to all $w^{j}(j=1, \cdots, m)$ so that identical weighting is applied to $F^{j}$. 


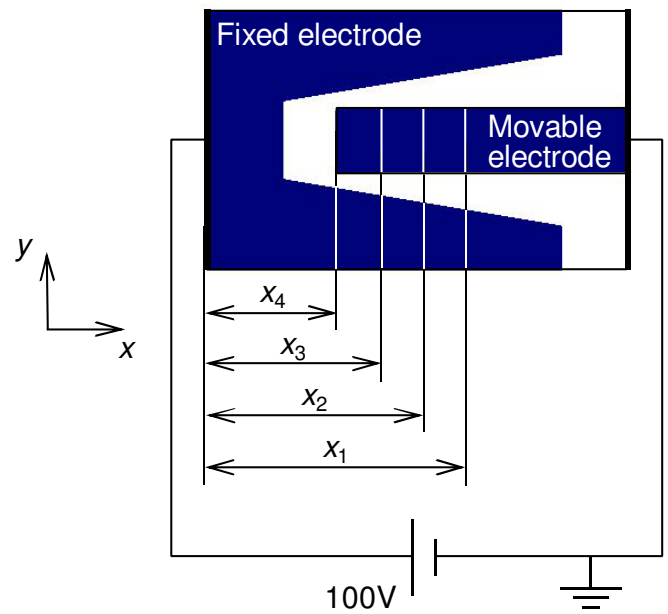

Fig. 8 Initial configuration and electrode's positions

\subsubsection{Linear driving force profile}

An driving force profile with a linear response rate is one desirable goal for the performance of electrostatic actuators. In the first and second examples, we obtain actuator designs that display a linear relationship between driving force and electrode position. The $x$ positions of the movable electrode in the first example are $x_{1}=400 \mu \mathrm{m}, x_{2}=333 \mu \mathrm{m}$, $x_{3}=267 \mu \mathrm{m}, x_{4}=200 \mu \mathrm{m}$, respectively, from the fixed electrode, as shown in Fig. 8.

Plots of the target driving force, initial driving force, and driving force for the optimal configuration are shown in Fig. 9 (a). We can confirm that the driving force profile of the optimal configuration is accurately aligned with the target driving force profile. The optimal configuration is shown in Fig. 10 (a). As shown in this figure, clear boundaries were obtained. The dotted lines in Fig. 10 (a) illustrate the four different $x$ positions of the movable electrode. Figure 11(a) shows the optimization history.

The second example also targets a linear driving force profile but uses five different movable-electrode positions in the model. The electrode positions here are $x_{1}=400 \mu \mathrm{m}$, $x_{2}=350 \mu \mathrm{m}, x_{3}=300 \mu \mathrm{m}, x_{4}=250 \mu \mathrm{m}, x_{5}=200 \mu \mathrm{m}$. The driving force profiles and optimal configuration are shown in Fig. 9 (b) and Fig. 10 (b), respectively. The driving forces obtained by the optimal configuration are congruent with the target driving force profile. Figure 11(b) shows the optimization history.

\subsubsection{Sharp initial rise driving force profile}

Most actuators used in engineering applications have a driving force profile with a weak initial rise. Since driving force profiles that include a sharp initial rise are advantageous in

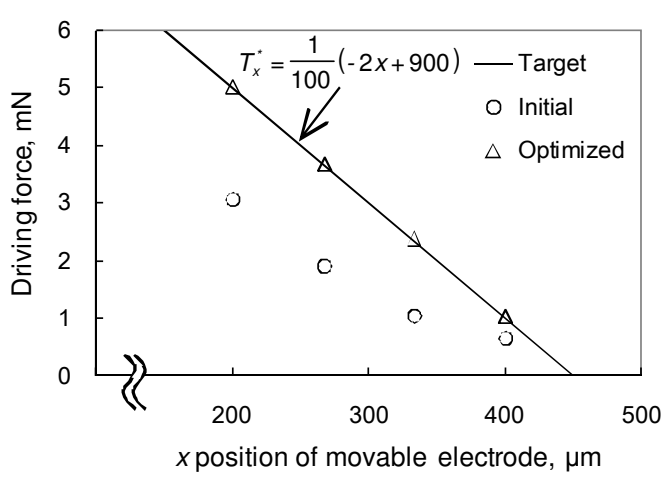

(a) Example 1

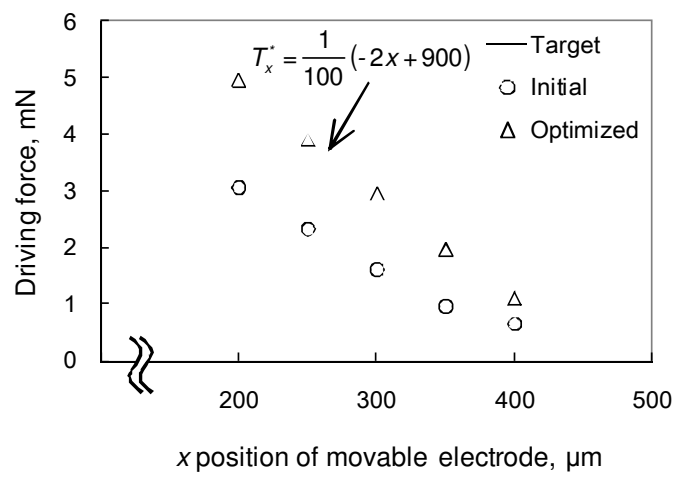

(b) Example 2

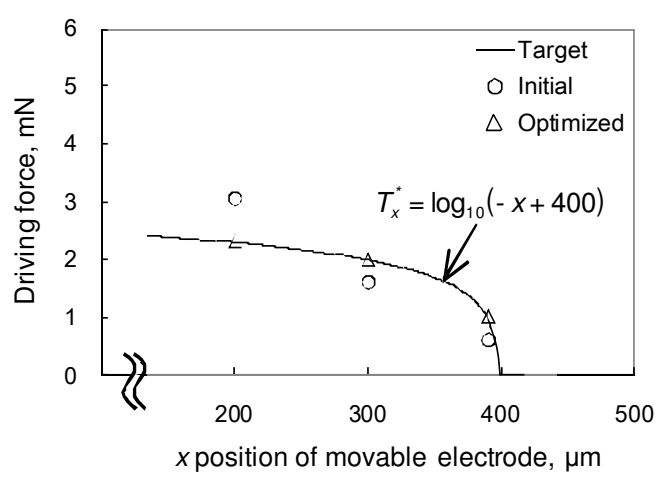

(c) Example 3

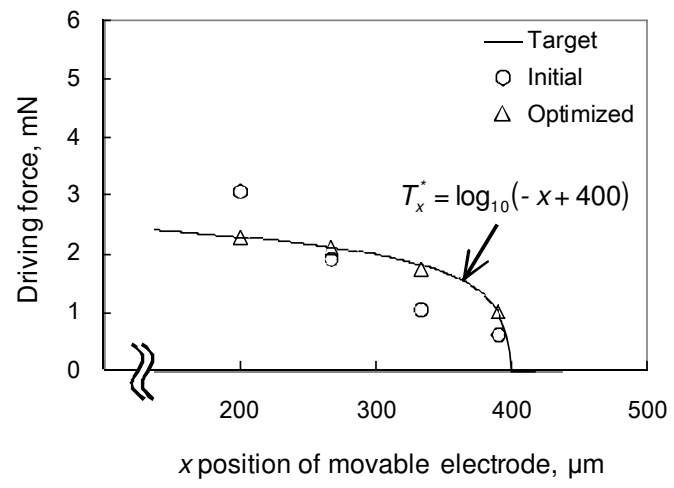

(d) Example 4

Fig. 9 Driving force profiles 


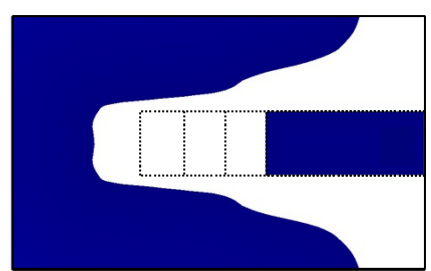

(a) Example 1

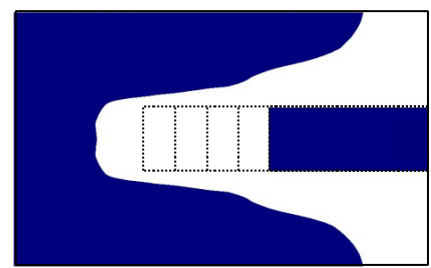

(b) Example 2

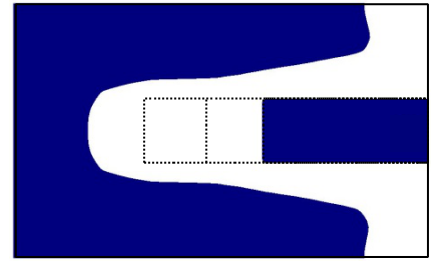

(c) Example 3

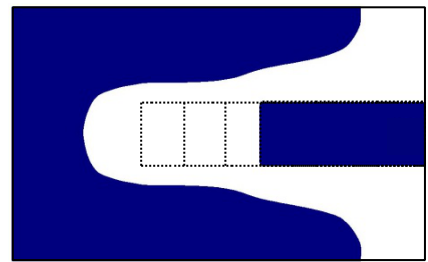

(d) Example 4

Fig. 10 Optimal configurations

industrial applications, we apply the proposed optimization method to the design of an actuator with this type of force profile.

In the third example, we set a target driving force profile that includes a sharp initial rise. As shown in Fig. 9 (c), the rate of increase in driving force is greatest when the electrodes are furthest apart, and this rate decreases as the electrodes approach. The model in this example uses three positions for the movable electrode, namely, $x_{1}=390 \mu \mathrm{m}$, $x_{2}=300 \mu \mathrm{m}$, and $x_{3}=200 \mu \mathrm{m}$. The driving force profile of the optimal configuration is shown in Fig. 9 (c). We can confirm that the optimal configuration obtains a driving force profile that matches the target driving force profile. The optimal configuration is shown in Fig. 10 (c). Figure 11(c) shows the optimization history.

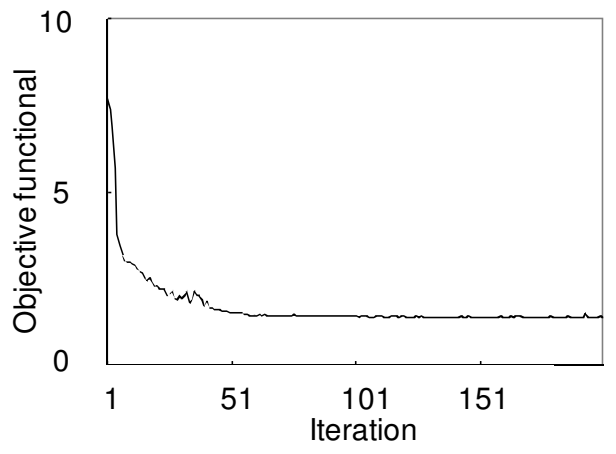

(a) Example 1

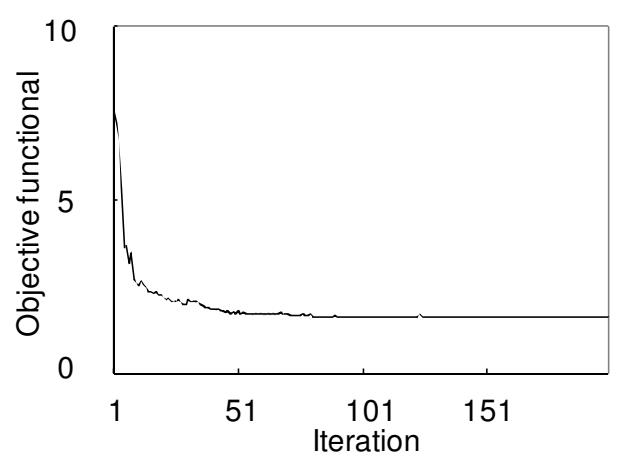

(b) Example 2

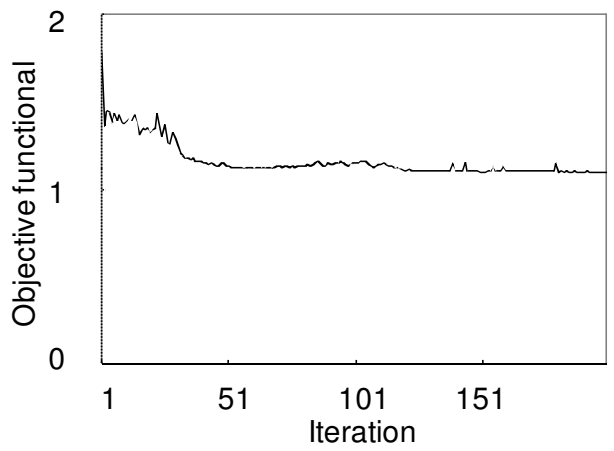

(c) Example 3

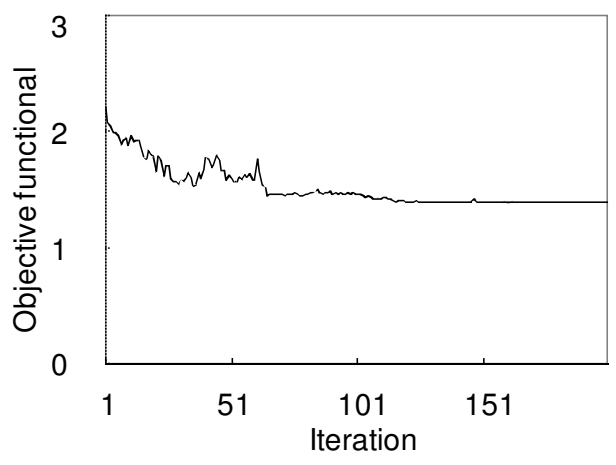

(d) Example 4

Fig. 11 Optimization history 
The fourth example also targets a sharp initial rise driving force profile but uses a model with four different electrode positions. The $x$ positions of the movable electrode in this example are $x_{1}=390 \mu \mathrm{m}, x_{2}=333 \mu \mathrm{m}, x_{3}=267 \mu \mathrm{m}$, and $x_{4}=200 \mu \mathrm{m}$, respectively. We can confirm that the driving force profile provided by the optimal configuration again matches the target driving force profile, as shown in Fig. 9 (d). The optimal configuration is shown in Fig. 10 (d). Figure 11(d) shows the optimization history.

\section{Conclusion}

This paper proposed a level set-based shape optimization method for comb drive electrostatic actuators. Our method enables actuator designs that achieve prescribed driving force profiles, which is highly beneficial in an engineering sense. We achieved the following:

(1) Design requirements of comb drive electrostatic actuators were clarified and an optimization problem using a multi-objective functional for an electrostatic comb drive type of actuator that achieves a prescribed driving force profile was formulated.

(2) The sensitivities were derived by the adjoint variable method and the objective functional we employed did not require solving the adjoint fields during the optimization.

(3) A mesh adaptation scheme for the optimization of the electrostatic actuators was developed, based on the level set method and a Laplacian smoothing method. A calculation scheme to accurately calculate the electrostatic forces on the electrode surfaces during the optimization was developed.

(4) Based on the formulation of the optimization problem and the mesh adaptation scheme, an optimization algorithm for solving the electrostatic field and calculating the sensitivities was constructed.

(5) The suitability of our proposed method was demonstrated in four numerical examples. Each optimal configuration achieved the target driving force profile, and all had clear structural boundaries. We confirmed that the proposed method enables the design of electrostatic actuators that incorporate desirable driving force profiles and obtains optimal configurations that are free from grayscale areas.

\section{Acknowledgments}

The first author gratefully acknowledges support from Yushin Precision Equipment Co., Ltd. The third author gratefully acknowledges support from JSPS KAKENHI Grant Number 24760065.

\section{Appendix: Sensitivity Analysis}

The details of the derivation of the sensitivity are now provided. First, the Lagrangian $\bar{F}$ is rewritten from (29) as follows:

$$
\begin{aligned}
\bar{F}= & \log \left[\sum_{j=1}^{m} \exp \left\{w^{j}\left(\int_{D} \boldsymbol{t}\left(\boldsymbol{\nabla} V^{j}\right) \overline{\boldsymbol{\delta}}(\boldsymbol{\phi}) \mathrm{d} D-\boldsymbol{T}^{j *}\right)^{2}\right\}\right] \\
& +\sum_{j=1}^{m}\left[-\int_{D}\left(\boldsymbol{\nabla} \hat{V}^{j}\right) \cdot\left\{\boldsymbol{\varepsilon}(\boldsymbol{\phi}) \boldsymbol{\nabla} V^{j}\right\} \mathrm{d} D+\int_{\Gamma} \hat{V}^{j} q^{j} \mathrm{~d} \Gamma\right]
\end{aligned}
$$

The Lagrangian $\bar{F}$ changes when the design variable $\phi$ slightly changes, so we have

$$
\begin{gathered}
\bar{F}+\delta \bar{F} \\
=\log \left[\sum _ { j = 1 } ^ { m } \operatorname { e x p } \left\{w ^ { j } \left\{\int_{D}\left(\boldsymbol{t}\left(\boldsymbol{\nabla} V^{j}\right)+\frac{\partial \boldsymbol{t}\left(\boldsymbol{\nabla} V^{j}\right)}{\partial\left(\boldsymbol{\nabla} V^{j}\right)} \boldsymbol{\delta}\left(\boldsymbol{\nabla} V^{j}\right)\right)\right.\right.\right. \\
\left.\left.\left.+\left(\overline{\boldsymbol{\delta}}(\phi)+\frac{\partial \overline{\boldsymbol{\delta}}(\phi)}{\partial \phi} \boldsymbol{\delta} \phi\right) \mathrm{d} D-\boldsymbol{T}^{j *}\right\}^{2}\right\}\right] \\
+\sum_{j=1}^{m}\left[-\int_{D}\left(\boldsymbol{\nabla} \hat{V}^{j}\right) \cdot\left\{\left(\varepsilon(\phi)+\frac{\partial \varepsilon(\phi)}{\partial \phi} \boldsymbol{\delta} \phi\right)\right.\right. \\
\left.+\int_{\Gamma} \hat{V}^{j}\left(q^{j}+\delta q^{j}\right) \mathrm{d} \Gamma\right]
\end{gathered}
$$

Therefore, we have

$$
\begin{aligned}
& \delta \bar{F}= \\
& \log \left[\sum _ { j = 1 } ^ { m } \operatorname { e x p } \left\{w ^ { j } \left\{\int_{D}\left(\boldsymbol{t}+\frac{\partial \boldsymbol{t}\left(\boldsymbol{\nabla} V^{j}\right)}{\partial\left(\boldsymbol{\nabla} V^{j}\right)} \boldsymbol{\delta}\left(\boldsymbol{\nabla} V^{j}\right)\right) \overline{\boldsymbol{\delta}}(\phi) \mathrm{d} D\right.\right.\right. \\
& \left.\left.\left.+\int_{D}\left(\boldsymbol{t}\left(\boldsymbol{\nabla} V^{j}\right) \frac{\partial \overline{\boldsymbol{\delta}}(\phi)}{\partial \phi} \boldsymbol{\delta} \phi\right) \mathrm{d} D-\boldsymbol{T}^{j *}\right\}^{2}\right\}\right] \\
& -\log \left[\sum_{j=1}^{m} \exp \left\{w^{j}\left(\int_{D} \boldsymbol{t}\left(\boldsymbol{\nabla} V^{j}\right) \overline{\boldsymbol{\delta}}(\boldsymbol{\phi}) \mathrm{d} \boldsymbol{D}-\boldsymbol{T}^{j *}\right)^{2}\right\}\right] \\
& +\sum_{j=1}^{m}\left[-\int_{D}\left(\boldsymbol{\nabla} \hat{V}^{j}\right) \cdot\left\{\varepsilon(\phi) \delta\left(\nabla V^{j}\right)+\frac{\partial \varepsilon(\phi)}{\partial \phi} \delta \phi \nabla V^{j}\right\} \mathrm{d} D\right. \\
& \left.+\int_{\Gamma} \hat{V}^{j} \delta q^{j} \mathrm{~d} \Gamma\right]
\end{aligned}
$$


where a second order variation is neglected. Here, following notations are introduced.

$$
\begin{aligned}
\boldsymbol{T}^{j}:= & \int_{D} \boldsymbol{t}\left(\boldsymbol{\nabla} V^{j}\right) \cdot \overline{\boldsymbol{\delta}}(\phi) \mathrm{d} D \\
\delta \boldsymbol{T}^{j} & :=\int_{D}\left(\frac{\partial \boldsymbol{t}\left(\boldsymbol{\nabla} V^{j}\right)}{\partial\left(\boldsymbol{\nabla} V^{j}\right)} \boldsymbol{\delta}\left(\boldsymbol{\nabla} V^{j}\right)\right) \overline{\boldsymbol{\delta}}(\phi) \mathrm{d} D \\
& +\int_{D}\left(\boldsymbol{t}\left(\boldsymbol{\nabla} V^{j}\right) \frac{\partial \overline{\boldsymbol{\delta}}(\phi)}{\partial \phi} \boldsymbol{\delta} \phi\right) \mathrm{d} D \\
W^{j}: & =\exp \left(w^{j}\left(\boldsymbol{T}^{j}-\boldsymbol{T}^{j *}\right)^{2}\right)
\end{aligned}
$$

Using the above notations, the first and second terms of the variation $\delta F$ can be evaluated as follows:

$$
\begin{array}{r}
\delta F=\log \left[\sum_{j=1}^{m} \exp \left\{w^{j}\left(\boldsymbol{T}^{j}+\delta \boldsymbol{T}^{j}-\boldsymbol{T}^{j *}\right)^{2}\right\}\right] \\
-\log \left[\sum_{j=1}^{m} \exp \left\{w^{j}\left(\boldsymbol{T}^{j}-\boldsymbol{T}^{j *}\right)^{2}\right\}\right] \\
=\log \left[\frac{\sum_{j=1}^{m} \exp \left\{w^{j}\left(\boldsymbol{T}^{j}+\delta \boldsymbol{T}^{j}-\boldsymbol{T}^{j *}\right)^{2}\right\}}{\sum_{j=1}^{m} \exp \left\{w^{j}\left(\boldsymbol{T}^{j}-\boldsymbol{T}^{j *}\right)^{2}\right\}}\right]
\end{array}
$$

Here, we recall the Maclaurin expansion with respect to $\exp (x)$ :

$\exp (x)=\sum_{n=0}^{\infty} \frac{x^{n}}{n !}$

Therefore, we have

$\delta F=\log \left[1+\frac{2 \sum_{j=1}^{m} w^{j} W^{j}\left(\boldsymbol{T}^{j}-\boldsymbol{T}^{j *}\right) \cdot \delta \boldsymbol{T}^{j}}{\sum_{j=1}^{m} W^{j}}\right]$

Furthermore, we recall the following relationship:

$e=\lim _{x \rightarrow 0}(1+x)^{\frac{1}{x}}$.

Therefore, we have

$\delta F=\left(\frac{2}{\sum W^{j}}\right) \sum_{j=1}^{m} w^{j} W^{j}\left(\boldsymbol{T}^{j}-\boldsymbol{T}^{j *}\right) \cdot \delta \boldsymbol{T}^{j}$

Given the boundary condition imposed on $\Gamma_{q}$, we have following relationship:

$\delta q^{j}=0 \quad$ on $\quad \Gamma_{q}$.

Thus, the boundary integral term can be evaluated as follows:

$\int_{\Gamma} \hat{V}^{j} \delta q^{j} \mathrm{~d} \Gamma \rightarrow \int_{\Gamma_{V}} \hat{V}^{j} \delta q^{j} \mathrm{~d} \Gamma$
The variation of $\bar{F}$ can therefore be evaluated as

$$
\begin{aligned}
& \delta \bar{F}=\sum_{j=1}^{m}\left[\int _ { D } \left[-2 \varepsilon_{0}\left\{\left\{\left(\nabla V^{j}\right) \otimes \overline{\boldsymbol{\delta}}(\phi)\right\}^{T} \boldsymbol{a}_{T}^{j}\right\}\right.\right. \\
& \left.\left.+\varepsilon_{0}\left\{\boldsymbol{a}_{T}^{j} \cdot\{\boldsymbol{I} \overline{\boldsymbol{\delta}}(\phi)\}\left(\boldsymbol{\nabla} V^{j}\right)\right\}-\varepsilon(\phi) \boldsymbol{\nabla} \hat{V}^{j}\right] \cdot \boldsymbol{\delta}\left(\boldsymbol{\nabla} V^{j}\right) \mathrm{d} D\right] \\
& +\sum_{j=1}^{m}\left[\int _ { D } \left\{\left(\frac{2 w^{j} W^{j}\left(\boldsymbol{T}^{j}-\boldsymbol{T}^{j *}\right)}{\sum W^{j}}\right) \cdot\left(\boldsymbol{t}\left(\boldsymbol{\nabla} V^{j}\right) \frac{\partial \overline{\boldsymbol{\delta}}(\phi)}{\partial \phi}\right)\right.\right. \\
& +\sum_{j=1}^{m}\left[\int_{\Gamma_{V}} \hat{V}^{j} \boldsymbol{\delta} q^{j} \mathrm{~d} \Gamma\right] .
\end{aligned}
$$

To make the terms pertaining to $\delta\left(\nabla V^{j}\right)$ and $\delta q^{j}$ equivalent to zero, we define the adjoint system as follows:

$$
\begin{cases}\nabla \hat{V}^{j}=-2 \frac{\varepsilon_{0}}{\varepsilon(\phi)}\left[\left\{\left(\boldsymbol{\nabla} V^{j}\right) \otimes \overline{\boldsymbol{\delta}}(\phi)\right\}^{T} \boldsymbol{a}_{T}^{j}\right] & \\ \quad+\frac{\varepsilon_{0}}{\varepsilon(\phi)}\left[\boldsymbol{a}_{T}^{j} \cdot\{\boldsymbol{I} \overline{\boldsymbol{\delta}}(\phi)\}\left(\boldsymbol{\nabla} V^{j}\right)\right] & \text { in } D \\ \hat{V}^{j}=0 & \text { on } \Gamma_{V}\end{cases}
$$

Then, $\delta \bar{F}$ can be expressed as

$$
\begin{gathered}
\delta \bar{F}=\sum_{j=1}^{m} \int_{D}\left\{\left(\frac{2 w^{j} W^{j}\left(\boldsymbol{T}^{j}-\boldsymbol{T}^{j *}\right)}{\sum W^{j}}\right) \cdot\left(\boldsymbol{t}\left(\boldsymbol{\nabla} V^{j}\right) \frac{\partial \overline{\boldsymbol{\delta}}(\phi)}{\partial \phi}\right)\right. \\
\left.-\left(\boldsymbol{\nabla} \hat{V}^{j}\right) \cdot\left(\frac{\partial \varepsilon(\phi)}{\partial \phi} \boldsymbol{\nabla} V^{j}\right)\right\} \delta \phi \mathrm{d} D .
\end{gathered}
$$

\section{References}

Allaire G, Jouve F, Toader A (2004) Structural optimization using sensitivity analysis and a level-set method. J Comput Phys 194(1):363-393

Alwan A, Ananthasuresh G (2006a) Coupled electrostaticelastic analysis for topology optimization using material interpolation. International MEMS Conference 2006, Journal of Physics Conference Series 34(1):264-270

Alwan A, Ananthasuresh G (2006b) Topology optimization of electrostatically actuated micromechanical structures with accurate electrostatic modeling of the interpolated material model. Proceedings of the ASME International Design Engineering Technical Conferences and Computers and Information in Engineering Conferences-2006

Bank RE, Smith RK (1997) Mesh smoothing using a posteriori error estimates. SIAM J on Numer Anal 34(3):979997 
Bank RE, Xu J (1996) An algorithm for coarsening unstructured meshes. Numerische Mathematik 73(1):1-36

Belytschko T, Black T (1999) Elastic crack growth in finite elements with minimal remeshing. Int $\mathbf{J}$ Numer Methods Eng 45(5):601-620

Bendsøe M (1989) Optimal shape design as a material distribution problem. Struct multidiscip Optim 1(4):193-202

Bendsøe M, Sigmund O (1999) Material interpolation schemes in topology optimization. Archive of Appl Mech 69(9):635-654

Bendsøe MP, Kikuchi N (1988) Generating optimal topologies in structural design using a homogenization method. Comput Methods Appl Mech and Eng 71(2):197-224

Buell WR, Bush BA (1973) Mesh generation - a survey. J Eng Ind 95(1):332-338

Dolbow J, Belytschko T (1999) A finite element method for crack growth without remeshing. Int $\mathrm{J}$ Numer Methods Eng 46(1):131-150

Field DA (1988) Laplacian smoothing and delaunay triangulations. Commun Appl Numer Methods 4(6):709-712

Grade J, Jerman H, Kenny T (2003) Design of large deflection electrostatic actuators. J Microelectromechanical Syst 12(3):335-343

Hirt C, Amsden A, Cook J (1997) An arbitrary LagrangianEulerian computing method for all flow speeds. J Comput Phys 135(2):203-216

Huerta A, Liu W (1988) Viscous flow with large free surface motion. Comput Methods Appl Mech and Eng 69(3):277-324

Kim J, Varadan V, Varadan V, Bao X (1996) Finite-element modeling of a smart cantilever plate and comparison with experiments. Smart Mater Struct 5(2):165-170

Legtenberg R, Groeneveld A, Elwenspoek M (1996) Combdrive actuators for large displacements. J Micromechanics Microengineering 6(3):320-329

Nemirovsky Y, Bochobza-Degani O (2001) A methodology and model for the pull-in parameters of electrostatic actuators. J Microelectromechanical Syst 10(4):601-615

Osher S, Fedkiw R (2003) Level Set Methods and Dynamic Implicit Surfaces. Springer-Verlag

Ou K, Chen K, Yang T, Lee S (2011) A novel semianalytical approach for finding pull-in voltages of micro cantilever beams subjected to electrostatic loads and residual stress gradients. J Microelectromechanical Syst 20(2):527-537

Qian X, Sigmund O (2013) Topological design of electromechanical actuators with robustness toward over-and under-etching. Comput Methods Appl Mech and Eng 253:237-251

Raulli M, Maute K (2005) Topology optimization of electrostatically actuated microsystems. Struct multidiscip Optim 30(5):342-359

Seeger J, Boser B (2003) Charge control of parallel-plate, electrostatic actuators and the tip-in instability. J Micro- electromechanical Syst 12(5):656-671

Sethian J (1999) Level set methods and fast marching methods: evolving interfaces in computational geometry, fluid mechanics, computer vision, and materials science, vol 3. Cambridge university press

Sethian J, Wiegmann A (2000) Structural boundary design via level set and immersed interface methods. J comput phys 163(2):489-528

Sukumar N, Chopp D, Moës N, Belytschko T (2001) Modeling holes and inclusions by level sets in the extended finite-element method. Comput Methods in Appl Mech Eng 190(46):6183-6200

Sussman M, Smereka P, Osher S (1994) A level set approach for computing solutions to incompressible twophase flow. Department of Mathematics, University of California, Los Angeles

Wang MY, Wang X, Guo D (2003) A level set method for structural topology optimization. Comput Methods Appl Mech and Eng 192(1-2):227-246

Yamada T, Izui K, Nishiwaki S, Takezawa A (2010) A topology optimization method based on the level set method incorporating a fictitious interface energy. Comput Methods Appl Mech and Eng 199(45):2876-2891

Yamada T, Izui K, Nishiwaki S (2011) A level set-based topology optimization method for maximizing thermal diffusivity in problems including design-dependent effects. J Mech Des 133(3)

Yamasaki S, Nishiwaki S, Yamada T, Izui K, Yoshimura M (2010) A structural optimization method based on the level set method using a new geometry-based re-initialization scheme. Int $\mathbf{J}$ Numer Methods Eng 83(12):1580-1624

Yamasaki S, Nomura T, Kawamoto A, Sato K, Nishiwaki S (2011) A level set-based topology optimization method targeting metallic waveguide design problems. Int $\mathbf{J} \mathrm{Nu}-$ mer Methods Eng 87(9):844-868

Yang RJ, Chuang CH (1994) Optimal topology design using linear programming. Comput Struct 52(2):265-275

Ye W, Mukherjee S (1999) Optimal shape design of threedimensional mems with applications to electrostatic comb drives. Int J Numer Methods Eng 45(2):175-194

Ye W, Mukherjee S, MacDonald N (1998) Optimal shape design of an electrostatic comb drive in microelectromechanical systems. J Microelectromechanical Syst 7(1):16-26

Yoon G, Sigmund O (2008) A monolithic approach for topology optimization of electrostatically actuated devices. Comput Methods Appl Mech and Eng 197(4548):4062-4075 